Laser Chem., 1999, Vol. 19, pp. 71-74

Reprints available directly from the publisher

Photocopying permitted by license only
(C) 1999 OPA (Overseas Publishers Association) N.V.

Published by license under

the Harwood Academic Publishers imprint,

part of The Gordon and Breach Publishing Group.

Printed in India.

\title{
SHORT-TIME PHOTODISSOCIATION DYNAMICS OF 1-CHLORO-2-IODOETHANE FROM RESONANCE RAMAN SPECTROSCOPY
}

\author{
XUMING ZHENG and DAVID LEE PHILLIPS* \\ Department of Chemistry, University of Hong Kong, Pokfulam Road, \\ Hong Kong
}

(Received 7 April 1997)

\begin{abstract}
We have obtained A-band absorption resonance Raman spectra of 1-chloro-2iodoethane in cyclohexane solution. We have done preliminary time-dependent wavepacket calculations to simulate the resonance Raman intensities and absorption spectrum in order to learn more about the short-time photodissociation dynamics. We compare our preliminary results for 1-chloro-2-iodoethane with previous resonance Raman results for iodoethane and find that there appears to be more motion along nonC-I stretch modes for 1-chloro-2-iodoethane than for iodoethane. This is consistent with results of TOF photofragment spectroscopy experiments which indicate much more internal excitation of the photoproducts from 1-chloro-2-iodoethane photodissociation than the photoproducts from iodoethane photodissociation.
\end{abstract}

Keywords: Resonance; Raman; 1-chloro-2-iodoethane; iodoethane; photodissociation

\section{INTRODUCTION}

Photodissociation of iodoethane leads to $32 \%$ to $39 \%$ of the available energy appearing in internal excitation of the photofragments while about $42 \%$ to $54 \%$ of the available energy goes into internal excitation of the 1-chloro-2-iodoethane photofragments [1,2]. We have obtained resonance Raman spectra of 1-chloro-2-iodoethane in its A-band absorption in order to elucidate the short-time photodissociation

${ }^{*}$ Corresponding author. 
dynamics that give rise to different degrees of internal excitation of the photofragments of 1-chloro-2-iodoethane compared to iodoethane.

\section{RESULTS AND DISCUSSION}

Figure 1 shows the A-band absorption spectrum of 1-chloro-2iodoethane in cyclohexane solution and Figure 2 shows the $266 \mathrm{~nm}$ resonance Raman spectrum of 1-chloro-2-iodoethane. For most 1, 2dihaloethanes, the trans conformation is the most stable in the gas phase and the gauche conformation is most stable in neat liquids [3, 4]. For most cyclohexane solutions of 1,2-dihaloethanes, the trans conformer in expected to be the most stable and have most of the relative population [5]. We have tentatively assigned most of the larger resonance Raman peaks to the trans conformer fundamentals, overtones, and combination bands of the nominal CCCI bend $\left(\nu_{10}\right)$, the nominal $\mathrm{C}-\mathrm{I}$ stretch $\left(\nu_{9}\right)$, and the nominal C $-\mathrm{CI}$ stretch $\left(\nu_{8}\right)$ modes. We have done preliminary time-dependent wavepacket calculations to simulate the resonance Raman intensities and

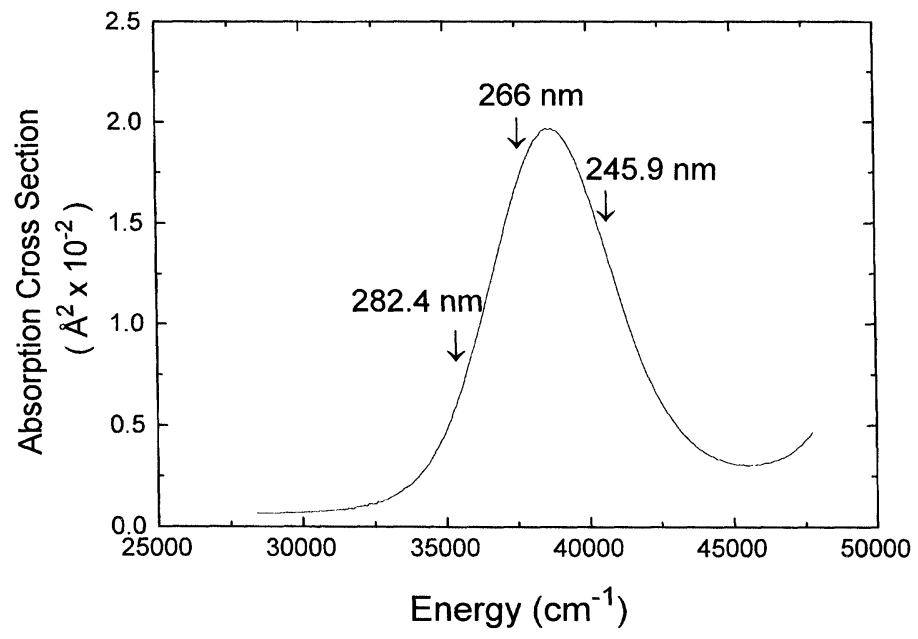

FIGURE 1 Absorption spectrum of 1-chloro-2-iodoethane in cyclohexane solution. The excitation wavelengths for the resonance Raman experiments are indicated by the numbers above the absorption spectrum. 


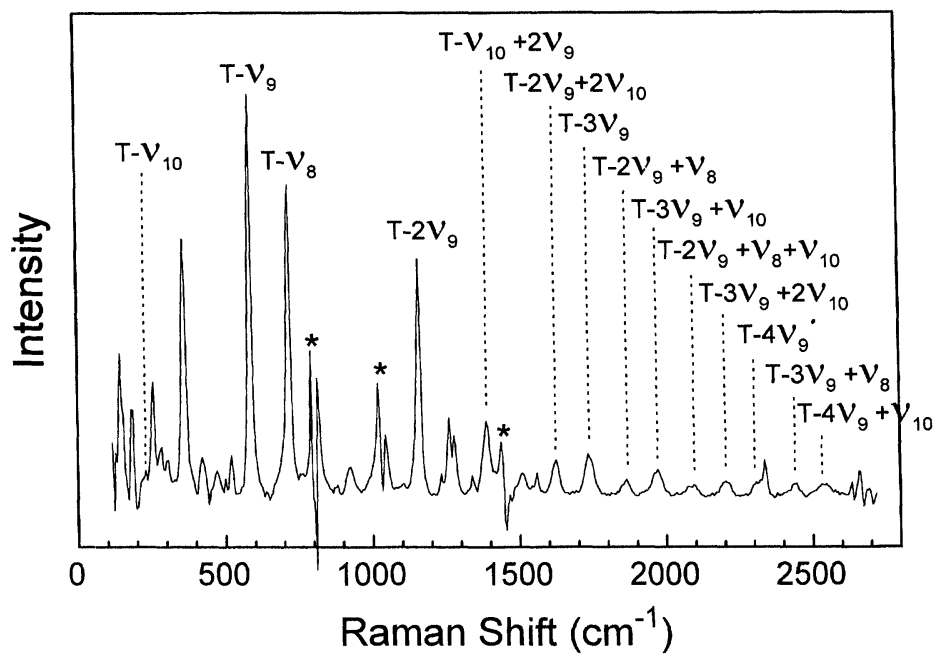

FIGURE 2 Resonance Raman spectrum of 1-chloro-2-iodoethane $(266 \mathrm{~nm})$. The larger Raman peaks tentatively assigned to the trans conformation are labeled (see text). The spectrum is intensity corrected and solvent substracted. The asterisks mark solvent subtraction artefacts.

absorption spectrum [6,7]. Table I presents the simulation best fit parameters for 1-chloro-2-iodoethane and those from a previous resonance Raman study for iodoethane [7]. Our results for 1-chloro-2iodoethane indicate that there are larger changes along non-C-I stretch modes relative to the nominal $\mathrm{C}-\mathrm{I}$ stretch mode in the Franck-Condon region than for iodoethane. This suggests that the short-time photodissociation dynamics of 1-chloro-2-iodoethane has larger geometry changes in the chloroethyl radical portion of the molecule as the $\mathrm{C}-\mathrm{I}$ bond breaks compared to changes in the ethyl radical part of iodoethane as the $\mathrm{C}-\mathrm{I}$ bond breaks. This is consistent

TABLE I Parameters for simulations of resonance raman intensities for: 1-chloro-2iodoethane (this work) and iodoethane (from Ref. [7])

\begin{tabular}{lcclcc}
\hline Mode & Frequency & $\Delta$ & \multicolumn{1}{c}{ Mode } & Frequency & $\Delta$ \\
\hline C-I stretch & $577 \mathrm{~cm}^{-1}$ & 4.50 & C-I stretch & $498 \mathrm{~cm}^{-1}$ & 4.50 \\
CCC1 bend & $232 \mathrm{~cm}^{-1}$ & 6.50 & CCI bend & $262 \mathrm{~cm}^{-1}$ & 3.00 \\
C-CI stretch & $707 \mathrm{~cm}^{-1}$ & 1.90 & $\mathrm{CH}_{3}$ rock & $1049 \mathrm{~cm}^{-1}$ & 0.35 \\
& & & $\mathrm{CH}_{2}$ wag & $1203 \mathrm{~cm}^{-1}$ & 0.60 \\
& & & $\mathrm{CH}_{3}$ antisym.def. & $1450 \mathrm{~cm}^{-1}$ & 0.50 \\
\hline
\end{tabular}


with the observation of more internal excitation of the photofragments of 1-chloro-2-iodoethane than of iodoethane [1,2]. Both increased mixing of $\mathrm{C}$ - I stretch internal coordinate in the normal mode descriptions and larger geometry changes in the Franck-Condon region may significantly contribute to the observed increase of intensity in nominal non-C-I stretch vibrational modes. Normal coordinate calculations and conversion of the normal mode displacements to internal coordinate motions at short-times $(<30 \mathrm{fs})$ are in progress. This will help to differentiate between these two effects and to determine more accurate short-time photodissociation dynamics.

\section{References}

[1] Paterson, C., Godwin, F. G. and Gorry, P. A. (1987). Molec. Phys., 60, 729.

[2] Minton, T. K., Felder, P., Brudzynski, R. J. and Lee, Y. T. (1984). J. Chem. Phys., 81, 1759.

[3] Liu, J. (1991). Ph.D. Dissertation, University of South Carolina.

[4] Durig, J. R., Liu, J. and Little, T. S. (1991). J. Phys. Chem., 95, 4664.

[5] Wiberg, K. R., Keith, T. A., Frisch, M. J. and Murcko, M. (1995). J. Phys. Chem., 99, 9072.

[6] Myers, A. B., In: Laser Techniques in Chemistry, Edited by Myers A. B. and Rizzo T. R. (Wiley, New York, 1995), p. 325.

[7] Phillips, D. L. and Myers, A. B. (1991). J. Chem. Phys., 95, 226. 